\title{
Autologous bone marrow concentrate enriched in progenitor cells - An adjuvant in the treatment of acute myocardial infarction
}

\author{
Vinay Sanghi a,b, Dalip Sethi ${ }^{c}$, Kenneth L. Harris ${ }^{c}$, Saniya Gupta ${ }^{c}$, Sheila Kar ${ }^{\text {d,e }}$, Mona Bhatia a , Upendra Kaul a , \\ Ashok Seth ${ }^{\mathrm{a}}$, Venkatesh Ponemone ${ }^{\mathrm{c}, *}$ \\ a Fortis Escorts Heart Institute and Research Centre, New Delhi, India \\ b Fortis Hospital Shalimar Bagh, New Delhi, India \\ c TotipotentRX Centre for Cellular Medicine (Cesca Therapeutics Inc.), Fortis Memorial Research Institute, Delhi (NCR), India \\ d Cedars Sinai Medical Center, Los Angeles, United States \\ e University of California, Los Angeles, United States
}

\section{A R T I C L E I N F O}

\section{Article history:}

Received 20 February 2016

Received in revised form 1 April 2016

Accepted 7 April 2016

Available online 18 April 2016

\section{Keywords:}

Acute myocardial infarction

AMIRST clinical trial

Cellular therapy

Intracoronary cell delivery

Point-of-care

STEMI

\begin{abstract}
A B S T R A C T
Despite advances in revascularization techniques, acute myocardial infarction (AMI) still carries significant morbidity and mortality. Over the past decade, the use of regenerative medicine methodologies, and specifically bone marrow derived progenitor cell therapy has been tested in more than 35 Phase I and Phase II clinical studies demonstrating overall safety and measurable clinical benefit, 12-61 months post-treatment as evaluated by improvement in the Left Ventricular Ejection Fraction (LVEF) and changes in infarct size post AMI. Recent meta-analysis on the subject highlighted several important parameters that include timing of the cell therapy post AMI, the cell dose, and the baseline LVEF on enrollment. We further postulate that the mythologies and timing for cell handling and delivery including the specific devices are essential for clinical efficacy. Addressing this we have developed a rapid 60 to 90 minute process and integrated system which is carried out in the heart catheter lab, using a combination product (U.S. Food and Drug broadly defined as the combination of co-labeled optimized "cell friendly" devices, effective cell/biological formulation and dose) for harvesting, processing, verifying, and delivering an autologous dose of bone marrow progenitor/stem cells via the intracoronary artery proximal to the infarct myocardial region. The methodology has been demonstrated to be safe and feasible for autologous in vivo use and presented by our groups' earlier studies ${ }^{1-3}$ and most recently used in a Phase Ib critical limb ischemia trial of 17 subjects (NCT01472289) (manuscript under preparation). This is the first case study prior to beginning the AMIRST trial [Acute Myocardial Infarction Rapid Stem cell Therapy], specific to our proprietary combination product kit for acute myocardial infarction, and was completed under the Independent Ethics Committee and Institutional Committee for Stem Cell Research and Therapy approval (TIEC/2011/32/ 02) for process and safety endpoints post-treatment.

(c) 2016 The Society of Cardiovascular Academy. Production and hosting by Elsevier B.V. All rights reserved. This is an open access article under the CC BY-NC-ND license (http://creativecommons.org/licenses/by-nc-nd/4.0/).
\end{abstract}

\section{Introduction and background}

Cell therapy is being widely explored in the management of various unmet medical need, and our own group has demonstrated safety and

\footnotetext{
* Corresponding author at: TotipotentRX Center for Cellular Medicine (Cesca Therapeutics Inc.), Fortis Memorial Research Institute, Sec 44, Gurgaon-122002, Delhi (NCR), India. Tel.: + 911244962251 (direct).

E-mail addresses: vinay.sanghi@fortishealthcare.com (V. Sanghi), dsethi@cescatherapeutics.com (D. Sethi), harriskl@me.com (K.L. Harris), s.gupta@cescatherapeutics.com (S. Gupta),skarmd@aol.com (S. Kar), monabhatia1@gmail.com (M. Bhatia), upendra.kaul@gmail.comc (U. Kaul), ashok.seth@fortishealthcare.com (A. Seth),vponemone@cescatherapeutics.com, ponemone@gmail.com (V. Ponemone).

Peer review under responsibility of The Society of Cardiovascular Academy.
}

efficacy of autologous bone marrow derived stem cells in treating various clinical indications. ${ }^{1-3}$ Cardiovascular disease (CVD) is the number one cause of morbidity and mortality worldwide. An estimated 17.3 million people died from CVDs in 2008, representing 30\% of all global deaths. ${ }^{4}$ Of these deaths, an estimated 7.3 million were due to coronary heart disease and 6.2 million were due to stroke. ${ }^{5}$ More remarkably, low- and middle-income countries are disproportionally affected, driving the need for regenerative therapies in lieu of chronic drug treatment regimens. Regenerative therapies must be offered in formats eliminating the need for high cost GMP laboratory infrastructure or extensive multi-hour usage of vascular catheter labs. Over $80 \%$ of CVD deaths take place in low- and middle-income countries and occur almost equally in men and women. ${ }^{4}$ In the progression of CVDs, plaque lesions develop in arteries that result in the narrowing of vessels, and in severe 
cases they break open and create a blockage of blood flow (ischemia) to vital parts of the heart. Such ischemia may be reversed if treated within a short period of time by reperfusion therapy, and further prevention of devastating remodeling is hypothesized by the infusion of adult tissuederived stem/progenitor cells. Despite significant advances in medical therapy and revascularization strategies, the prognosis of certain patients with acute myocardial infarction (AMI) remains dismal without the introduction of early biological repair intervention.

Along with reperfusion, adjuvant progenitor cell therapy has been shown to be potentially efficacious in the repair and regeneration of damaged heart tissue. These potent progenitor cells can be isolated from different sources within the adult human body. Specifically, the current cardiac regenerative field is experiencing diverse adult stem/ progenitor cell clinical trials at different stages of clinical development including bone marrow derived mononuclear cells (BMMNCs), ${ }^{6-8}$ mesenchymal stem cells (MSCs), ${ }^{9}$ adipose tissue-derived stem cells, ${ }^{10}$ and cardiac-derived expanded stem cells. ${ }^{11}$ Of the above mentioned cellular populations, bone marrow derived mononuclear cells have received greater scientific and translational attention. MSCs, although easily expanded in a laboratory setting and creating the ideal off-the-shelf cellular product, can generate local immune responses and disturb homeostasis within a tissue environment by releasing inflammatory mediators. ${ }^{12}$ Autologous bone marrow derived stem cell (aBMMNCs) therapy has emerged as a novel approach to treat patients with residual left ventricular dysfunction following AMI despite successful revascularization. ${ }^{7,38}$ Autologous sources are preferable because immunologic rejection is avoided by default. ${ }^{39}$ The first clinical trial evaluating the effects of stem cell therapy on ischemic heart failure was reported in 2002. In 2006, three large randomized clinical trials (ASTAMI, REPAIRAMI, and TOPCARE-CHD) were published. ${ }^{40-42}$ Although results on the beneficial effects of stem cells on myocardial function from these first trials were somewhat conflicting, further clinical trials (BOOST, STARHEART, SCIPIO, CADUCEUS, REGENT, FocusHF, and others) suggest the beneficial effects of stem cells on the function and remodeling of ischemic myocardium. ${ }^{43-47}$ Autologous BMMNCs not only circumvent the ethical and legal issues related to embryonic stem cells, but also overcome the risk of transmitting diseases and immune rejection. In last 12 years, autologous BMMNCs has been extensively studied for cardiac repair and regeneration in a number of randomized-controlled trials (RCTs). More than 1800 subjects have been investigated in these studies, and there is a clear evidence for safety of this therapy, however, its efficacy is still debated because of inconsistent results reported in the literature. ${ }^{27}$ Here we are presenting a case report utilizing our integrated treatment platform (combination product) that is specific for Acute Myocardial Infarction and has appropriately considered the essential devices, diagnostics, cell formulations, and instructions for use ensuring the treatment meets the objectives of providing a safe, effective, rapid, bedside therapy for treating low ejection fraction primary acute myocardial infarction.

\section{Case report}

\section{Case presentation}

A 43 year old male, non-diabetic, normotensive, non-obese, smoker presented with a history of two hours of chest pain and symptomatic of an AMI into the emergency department. On admission, the patient presented with a $2 \mathrm{~mm}$ ST segment elevation in V1 and V2 anterior leads with on-going chest pains, and AMI was further confirmed with biochemical blood tests. The patient's Left Ventricular Ejection Fraction (LVEF), was estimated to be around 35\% by bedside 2D ECHO. Diagnostic coronary angiography showed 95\% occlusion in the LAD in the proximal segment, and no collaterals were noted. RCA, and circumflex arteries were patent. Primary percutaneous coronary intervention (PCI) was performed within $24 \mathrm{~h}$ of onset of chest pains using a routine technique, and a single drug-eluting stent was deployed in the proximal LAD with
TIMI-3 grade flow results. Post-PCI, the patient's LVEF remained $<40 \%$ at the $120 \mathrm{~h}$ time-point as measured by multigated acquisition (MuGA) and ECHO, which met our inclusion criteria and is predictive of a higher than acceptable one year mortality rate. Also, the ECG showed T-wave inversion in anterior leads and resolution of J-point elevation post-PCI.

The patient was advised that he met the inclusion criteria for the AMIRST clinical trial program using his own (autologous) bone marrow progenitor cells. The clinical trial is registered with clinicaltrials.gov (NCT01536106) and is approved by the Institutional Ethics Committee (IEC) (IEC Approval \#TIEC/2011/32/02) and Institutional Committee for Stem Cell Research (ICSCRT). The Patient, Primary Investigator and Clinical Investigator concurred, and consent was obtained. On the sixth day post PTCA/stent implant, the patient was transferred to the heart catheterization laboratory, and the AMIRST (Acute Myocardial Infarction Rapid Stem cell Therapy) protocol was completed. The entire procedure was completed within $90 \mathrm{~min}$, in the catheterization laboratory using our point-of-care technology. As a preliminary safety study prior to full subject enrollment, the patient was followed up for 24-months, and evaluated with standard diagnostic metrics. No major adverse cardiac events (MACE) including re-hospitalization were reported during the 24 month follow-up period, demonstrating the preliminary safety of the device output, that is, autologous bone marrow cell concentrate enriched progenitor cells and the corresponding adjuvant treatment. The patients' LVEF improved from 35\% (Day 0 ) at the time of the AMIRST treatment to $60.3 \%$ following 24 months post-AMIRST intervention. It is noted that although the authors believe cardiac MRI is now the gold standard for measuring LVEF; in this study we used MuGA as the enrollment measurement technique. Caution should be taken in comparing MuGA and MRI LVEF results; however the enrollment LVEF was confirmed via a secondary method i.e. ECHO and the 3-month and 24-months LVEF results were confirmed by the same radiology team.

\section{Method: BMCePC adjuvant therapy}

Upon written informed consent, and within our maximum window of 10 days post MI, the patient was taken to the heart catheterization laboratory (operating room suite) on Day 6 post-PCI; mildly to moderately sedated using $0.2 \mathrm{mcg} / \mathrm{kg}$ of Fentanyl, and $120 \mathrm{~mL}$ of bone marrow was aspirated from the patient's iliac crest using an 11-gauge Jamshidi needle optimized for cell harvest. Careful bone marrow aspiration technique was employed to reduce peripheral blood contamination in the aspirate. Following the aspiration, the bone marrow was processed employing our proprietary point-of-care technology platform to produce bone marrow concentrate enriched in progenitor cells (BMCePC). Our point-of-care technology processes autologous bone marrow to a pre-determined multi-phasic concentrate effectively and safely in minimal time ex-vivo within 90 min (from aspiration to delivery) in the operating room. The technology employs a linked two phase stratification process using optical sensors to detect the different cell strata and additional sensors to detect the mass and volume. The point-ofcare device automatically separates the different cellular fractions into their designated compartments. Our point-of-care technology is a completely closed sterile system with minimal manipulation of the cells, that is, no addition of xenogeneic or chemical substance at any step from harvest to delivery (except anticoagulant) achieving at least $85 \%$ of cell yield. Rapid point-of-care quality testing instruments have been optimized and are used to ensure a safe and consistent cell dose. The cellular product obtained after processing the bone marrow using our device contained a total of $3.54 \times 10^{8}$ BMMNCs. A guide-wire was introduced into the femoral artery followed by a double lumen ultralow profile PTA intracoronary catheter, and the patient had four separate induced ischemia/progenitor cell infusions using the "stop-flow" technique before the entire optimal dose of nucleated cells was distally delivered to the stent in the LAD. The complete process was accomplished in 90 min at patient's bedside. The patient's hematological and 
Table 1

Clinical laboratory values pre- and post-BMCePC therapy

\begin{tabular}{lcll}
\hline & $\begin{array}{l}\text { Pre-BMCePC } \\
\text { infusion }\end{array}$ & $\begin{array}{l}\text { Post-BMCePC } \\
\text { infusion }\end{array}$ \\
& Day -2 & & Day 0 \\
\hline Hemoglobin $(\mathrm{g} / \mathrm{dL})$ & 13.5 & 13.8 \\
RBC count $\left(\times 10^{6} / \mu \mathrm{L}\right)$ & 4.84 & & 4.92 \\
Platelet count $\left(\times 10^{3} / \mathrm{mL}\right)$ & 110 & & 120 \\
Creatinine, serum $(\mathrm{mg} / \mathrm{dL})$ & 0.8 & & 0.9 \\
Urea nitrogen $(\mathrm{mg} / \mathrm{dL})$ & 13 & & 12 \\
Creatine kinase, serum & 106 & & 188 \\
N-terminal pro-B type natriuretic peptide $(\mathrm{pg} / \mathrm{mL})$ & 731.3 & & - \\
\hline
\end{tabular}

biochemical parameters are listed in Table 1. There were no adverse events $(\mathrm{AE})$ or serious adverse events $(\mathrm{SAE})$ reported during the procedure. The patient remained hospitalized for telemetry an additional 24 hours post cell transplant, and released with standard cardiac therapeutics as listed in Table 2.

\section{Patient follow-up}

The patient was scheduled for follow-up on 1, 2, 3, 6, 12 and 24 months to assess the primary endpoints of safety and the secondary endpoints of efficacy that include LVEF, MACE, cardiac remodeling and quality of life assessments. The 12-month follow-up could not be completed due to non-availability of patient, but all other follow-up points were completed. No Major Adverse Cardiac Events (MACE) or re-hospitalization events were reported. The patient continued to follow a normal life, after 2 weeks post the AMIRST procedure. At the 1-month follow-up, HOLTER monitoring was performed for $23 \mathrm{~h}$ and $6 \mathrm{~min}$. No ventricular ectopic were observed, and the heart variability was normal. The slowest episode of bradycardia (HR $53 \mathrm{bpm}, 1 \mathrm{~min}$ $13 \mathrm{~s}$ ) was observed at midnight and the fastest episode of tachycardia (HR $141 \mathrm{bpm}, 1 \mathrm{~min} 2 \mathrm{~s}$ ) was observed in the afternoon. Cardiac imaging was performed at each follow-up. The cardiac chambers appeared normal with no signs of pericardial effusion.

Overall, the study demonstrated preliminary safety of our bone marrow aspiration, processing and infusion methodology in an acute low LVEF infarct patient post PTCA. Fig. 1 shows cardiac MR images obtained 3 and 24-months post-BMC infusion. At the 24-month follow-up, the cardiac MR findings were summarized as "Basal and mid-cavity anterior and anteroseptal and apical anterior and septal and apex myocardial post contrast sub-endocardial $<25 \%$ to $50 \%$ with a focal spec of $75 \%$ transmural hyper enhancement, consistent with ischemic infarction."

The pre-BMC infusion cardiac MR imaging was performed on a different instrument than the 3- and 24-months post-BMC infusion. Therefore, an absolute quantitative equivalency measurement of LVEF between the pre-treatment and 3-months post-treatment was evaluated cautiously. Also, the MuGA and ECHO scan results have a level of user-dependency, and each result was cautiously interpreted. ${ }^{13,14} \mathrm{Nev}$ ertheless, a considerable improvement in the LVEF has been noted over the study period and between 3-months and 24-months follow-up, post-BMC infusion, the LVEF further improved from $55.4 \%$ to $60.3 \%$ as shown in Table 3. This degree of improvement is considered atypical for a patient having suffered an ST elevated myocardial infarction with an ejection fraction below $40 \%$ post reperfusion (stenting). The cardiac output (volumetric) also showed an improvement from $2.7 \mathrm{~L} / \mathrm{min}$ to

Table 2

Medications prescribed on discharge post-BMCePC therapy.

\begin{tabular}{llll}
\hline Drug & Dose & Frequency & Drug class \\
\hline Aspirin & $150 \mathrm{mg}$ & Oral, once daily & Anti-platelet \\
Copidogrel & $75 \mathrm{mg}$ & Oral, twice daily & Anti-platelet \\
Rosuvastatin & $20 \mathrm{mg}$ & Oral, once daily & Statin \\
Pantoprazole & $40 \mathrm{mg}$ & Oral, once daily & Dyspepsia/Antacid \\
\hline
\end{tabular}

$3.4 \mathrm{~L} / \mathrm{min}$ over the same period, and is a secondary endpoint. No reduction in scar size was observed with a heart mass of $115.5 \mathrm{~g}$ and a scar mass of $11.5 \mathrm{~g}$ (approximately $11 \%$ ).

\section{Discussion}

The AMIRST trial has been designed to determine the safety and preliminary effectiveness of our patented point-of-care technology (combination product) for aspiration, processing and intracoronary administration of BMCePCs for treatment of acute ST segment elevation MI with low ejection fraction. Here we are presenting the preliminary safety and effectiveness data from a single case prior to starting an elaborate, randomized-controlled AMIRST trial on a larger patient population. The results from this case study demonstrate that our combination product which is the first of its kind point-of-care technology that includes all the devices necessary for aspiration, processing and delivery of BMCePCs and also, checks the quality and quantity of the cells prior to administration using our optimized point-of-care diagnostic devices is efficacious for cardiac disorders in terms of safety and feasibility. We observed no significant major adverse effects in terms of MACE or pro-arrhythmia following administration of BMCePCs, and there was significant improvement in myocardial perfusion following administration of BMCePCs as determined by cardiac MRI, MuGA and 2D ECHO. Compared to baseline, there was a significant improvement in LVEF at 3 and 24 months in the treated patient. However, there was no significant improvement in scar size after administration of BMCePCs. Nevertheless, this case study was designed to demonstrate the preliminary safety and effectiveness data for our rapid bedside point-of-care technology (combination product) in the treatment of low ejection fraction AMI.

The adult human body possesses the potential to repair damaged cardiac tissue, and in the last decade considerable attempts have been made to harness this intrinsic regenerative capability. Adult human bone marrow represents the richest source of multi-potency progenitor cells. These progenitor cells can be harvested, processed/expanded and applied to regenerative applications. Despite obtaining excellent results in lab animal studies, human clinical results have remained underwhelming in the prior studies. The review of current and past literature, recent meta-analysis and reviews, all revealed critical stepwise procedural, instrumentation, and chemical/biological variables that should have been controlled and measured in order to ensure proper cardiac repair and regeneration in everyday interventional cardiology settings. Simply assuming specific cell types demonstrated in a lab model are ready for clinical trial, and expectations that such biology alone is predictable and reproducible equates to the pharmaceutical industry completely skipping the quality demands and parameters of statistical process control in manufacturing.

The main finding of the present study was that intracoronary cell therapy after AMI was potentially safe and resulted in a modest yet significant increase in LVEF. LVEF is one of the key indications of mortality rates post MI with a reduced LVEF being a risk factor for both sudden and non-sudden death, with the odds ratio for 1-year mortality after MI at 9.48 for patients with LVEF $\leq 30 \%$ compared with patients with LVEF $>50 \%, 2.94$ for patients with LVEF $30-40 \%$, whereas the risk was not significantly increased in patients with LVEF 40-50\%. Intracoronary cell therapy helps improve myocardial functions, however the exact mechanism of action is still debatable. Several hypothesis have been proposed and a recent well-conducted study suggested that bone marrow derived progenitor cells do not transdifferentiate into cardiomyocytes, instead they adopt mature hematopoietic characteristics. ${ }^{29,30}$ However, adult CD34 + cells can differentiate into cardiomyocytes, mature endothelial cells and smooth muscle cells in vivo. ${ }^{31}$ Another proposed mechanism is that, following cell therapy angiogenesis increases, thus, improving blood supply to the ischemic regions, which can potentially aide in revascularization of hibernating myocardium ${ }^{32}$ and inhibit cardiomyocyte apoptosis. ${ }^{33}$ The alternate possible reasons for LV recovery 

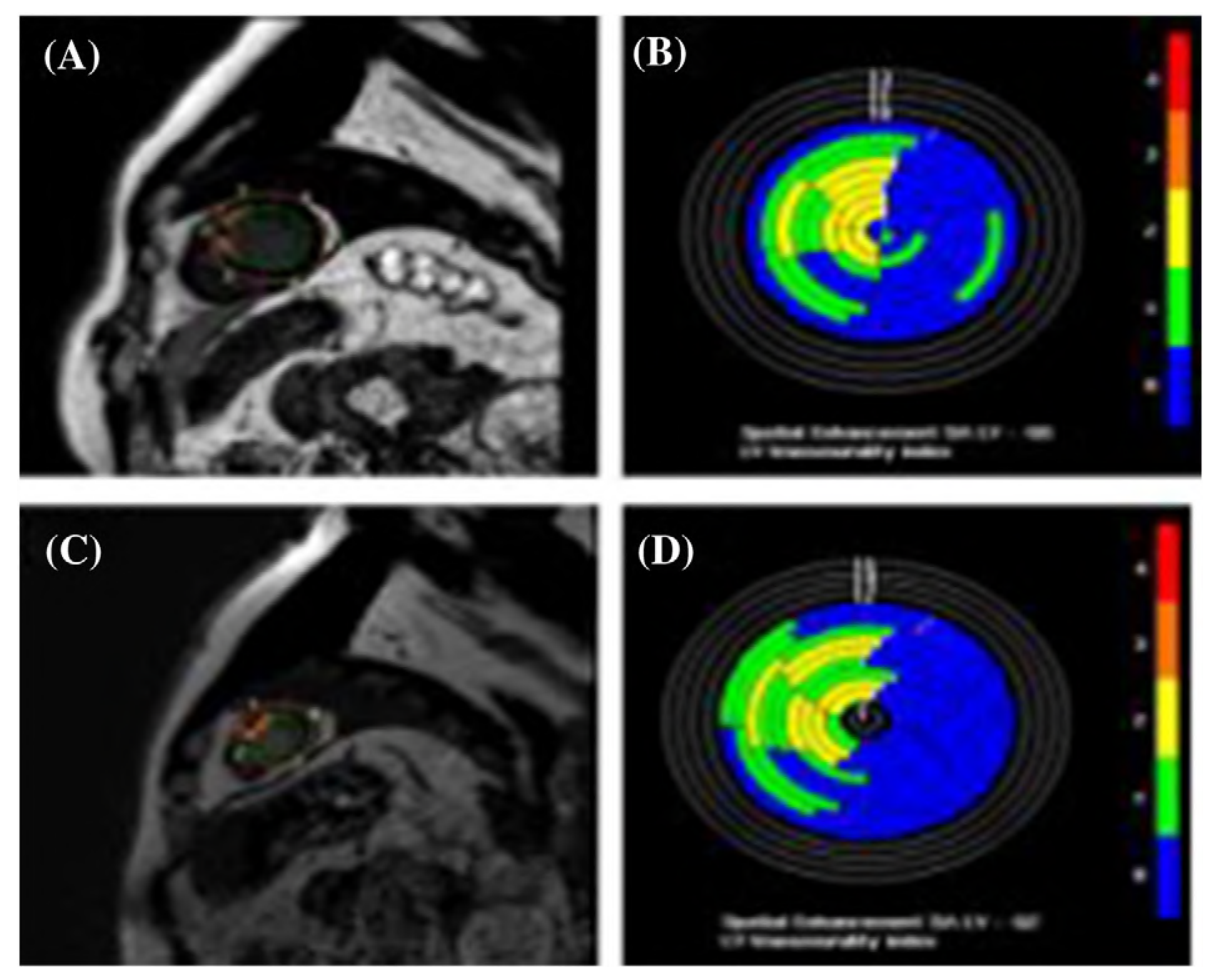

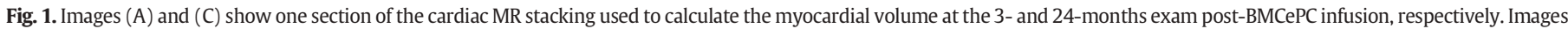
(B) and (D) represent the LV transmurality index at 3- and 24-months post-BMCePC infusion, respectively.

could be due to absence of co-morbidities, normal after load and early presentation of the patient. ${ }^{34}$

The regenerative potential of bone marrow derived cellular products is highly influenced by the handling, aspiration, processing, and re-delivery techniques as well as the chemistry, biology and timing of the cellular product intervention. It has been extensively reported and is well understood that the chemokine receptor-4 (CXCR4)/stromal cell-derived factor-1 (SDF-1) axis plays a crucial role in homing to and engraftment of progenitor cells to the heart after myocardial infarction (MI). ${ }^{15,16}$ Within minutes following a myocardial infarction, the cardiac tissue turns hypoxic leading to cardiac myocytes apoptosis and upregulation of several important factors, especially the stromal cell-derived factor-1 (SDF-1) chemokine. The increased levels of SDF-1 lead to homing of endogenous and bone marrow derived progenitor cells including endothelial progenitor cells (EPCs) that play a pivotal role in angiogenesis (formation of blood vessels). The progenitor cells that express CXCR4 respond swiftly to an increased SDF-1 gradient. Although, progenitor cells present in the bone marrow do express CXCR4 receptors, they need to mobilize or egress into the blood stream to efficiently perform the cardiac repair. It has been suggested in prior publications that bone marrow pro-genitor cells become more responsive to SDF-1 over 4 to 7 days post MI, however, the local (heart organ) cardiac SDF-1 levels decline quickly within 4 to 7 days from the cardiac injury (MI) leading to ineffective prolonged homing of progenitor cells to the injured tissue. ${ }^{17}$ Therefore, harvesting these potentially SDF-1 responsive, CXCR4 expressing bone marrow stem/progenitor cells, and infusing

Table 3

Time endpoints: left ventricular ejection fraction (LVEF) values and safety.

\begin{tabular}{llcl}
\hline Measurement time & Method & Value & MACE \\
\hline Time 0 days & 2D echo & $35 \%$ & $\mathrm{~N} / \mathrm{A}$ \\
Time 6 days & MuGA & $<40 \%$ & $\mathrm{~N} / \mathrm{A}$ \\
Time 7 days & IC angiogram & $<40 \%$ & None \\
Time 3 months F/U & cMRI & $55.30 \%$ & None \\
Time 24 months F/U & cMRI & $60.30 \%$ & None \\
\hline
\end{tabular}

them locally (proximally) to the stunted or scarred cardiac tissue represents a logical and viable approach for cardiac regenerative therapy. Fig. 2 represents an overview of the approach.

The importance of the CXCR4/SDF-1 axis is unambiguous in the arena of regenerative medicine, and chemicals that interfere with this mechanism definitely impact the over-all efficacy of the infused cellular product. Anticoagulants, a chemical added to bone marrow aspirate to prevent the formation of microthrombae, plays a crucial role in the overall efficacy of regenerative cell therapy. It is understood that the addition of any chemical entity in the presence of proteins or cells may have an effect on their structure or function or both. Heparins are the most commonly employed anticoagulants for bone marrow aspiration, and are reported to disrupt the pivotal CXCR4/SDF-1 axis ${ }^{18}$ and immunomodulation. ${ }^{19}$ Heparins have a high affinity for SDF-1 (Kd $22.7 \mathrm{nM}$ ), and bind to SDF-1 by electrostatic interactions that can inhibit its receptor (CXCR4) interactions. ${ }^{16}$ The heparin-treated bone marrow cells become unresponsive due to inhibition of the CXCR4 receptor internalization that further blocks CXCR4 downstream signaling.

It has also been reported that circulating VEGF levels, a potent proangiogenic factor, decrease by $93.2+5 \%$ within 30 min of unfractionated heparin (UFH) therapy. ${ }^{22}$ The decrease in circulating VEGF levels could be due to sequestration of VEGF into the extracellular matrix. Moreover, heparins are associated with high rates of preprocedural bleeding, which may be related to their inability to bind to clot-bound thrombin. Heparins can bind to platelet factor- 4 in vivo that may lead to formation of antibodies against the heparin/PF-4 complex and can cause heparin induced thrombocytopenia (HIT).$^{20}$

Williams et al. demonstrated that the presence of antibodies to the platelet factor-4/heparin complex serves as an independent predictor of myocardial infarction at 30 days in patients presenting with acute coronary ischemic syndromes. ${ }^{21}$ Therefore, our first variable control that we have devised and utilized in the AMIRST procedure and combination product kit is a novel anticoagulation methodology utilizing a U.S. FDA approved short synthetic peptide that can keep the aspirated bone marrow in a non-coagulated state, during processing and infusion, without affecting the biological efficacy of cellular product. 


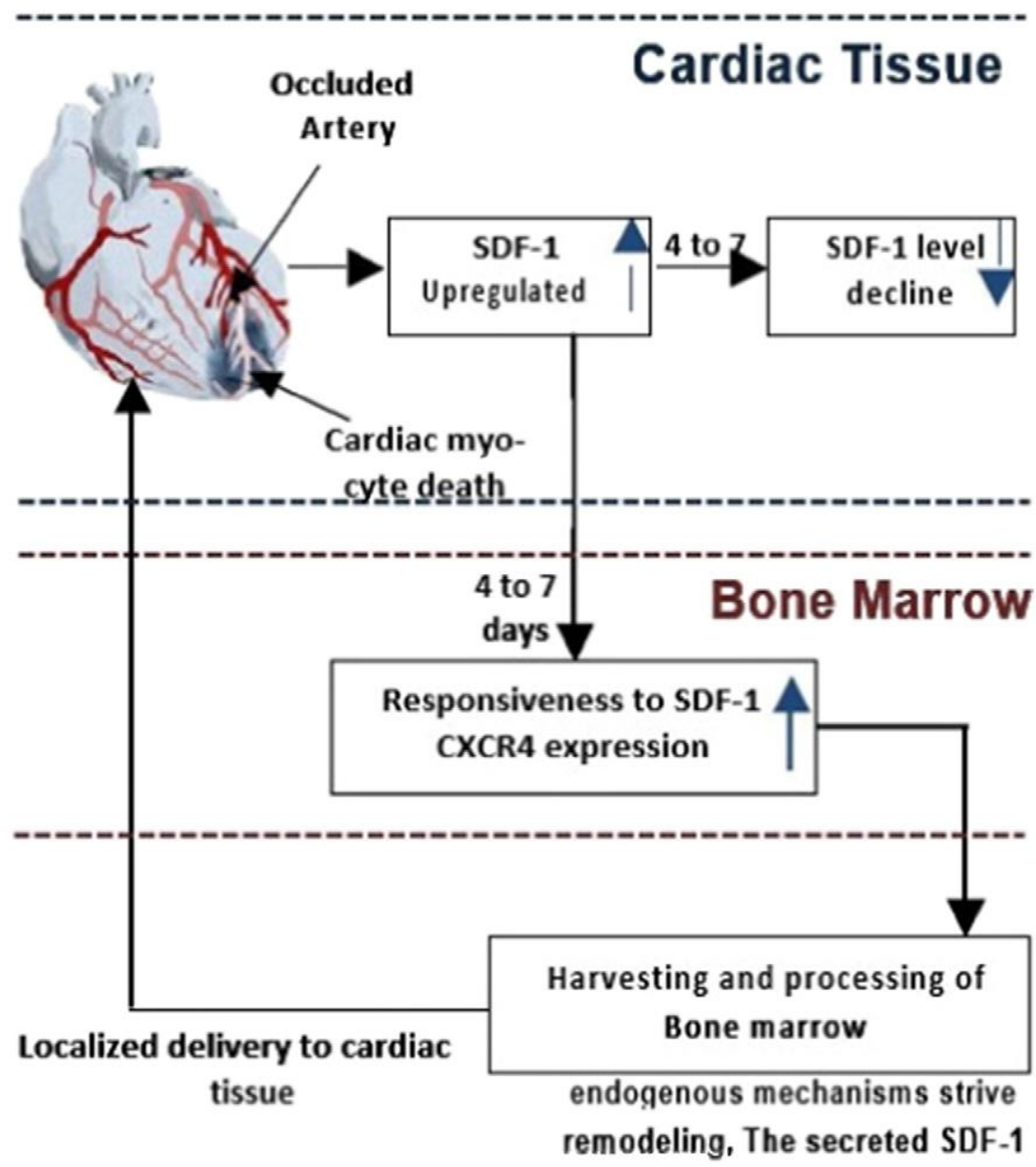

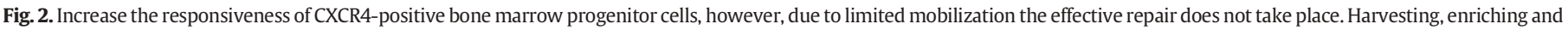
infusing potential progenitor cells are our approach for cardiac repair and regeneration.

Another major factor that influences the potency of bone marrow derived cellular product is the processing technology employed to harvest the desired cellular fraction(s). Some scientists and clinicians suggest that different processing techniques utilized in bone marrow processing, at least to some extent, are a plausible reason for conflicting and unpredictable results in clinical trials. ${ }^{22}$ It has been reported in the literature that efficacy and functionality of bone marrow derived cellular product is significantly influenced by various factors such as red blood cell contamination, ${ }^{23}$ content of apoptotic cells, washing steps, and inclusion of neutrophils. Till date, most clinical and pre-clinical studies have used the Ficoll-paque density gradient method to extract the cell fraction enriched with mononuclear cells, and most recently the TIME randomized trial ${ }^{24}$ used such a combination of automation and Ficoll-paque and reported consistently low total nucleated cell recoveries. Manual cell preparation studies have reported that ficollpaque methods result in a mere 15 to $30 \%$ recovery rates of bone marrow mononuclear cells following a multi-hour laboratory processing requirement. ${ }^{25}$ The lower recovery rates of ficoll-paque may among others be a consequence of density-medium related cytotoxicity. Moreover, the manual ficoll-paque method is highly user-dependent but should have been addressed in the automated approach used in the TIME study. This is likely another plausible reason for inconsistent results in clinical trials thus far. ${ }^{28}$ We have thus focused on critical variable number two by developing an automated chemical free "intelligent" cell-processing technology with Thermogenesis Corporation (USA) that yields reproducible cellular product and is independent of the user, assuming basic training. The method produces autologous
BMCePC, without the addition of density grade medium, at the patient's bedside in under $30 \mathrm{~min}$. Additionally, a clinical team using the AMIRST approach is able to verify that the harvesting and processing steps have yielded the desired minimum cellular dose by employing our rapid bedside diagnostics.

Handling time is a critical parameter in cellular therapy and the time interval between bone marrow aspiration and delivery of cellular product must be reduced. It has been suggested that bone marrow derived autologous cellular products prevent adverse cardiac remodeling by a synergy of mechanisms as described in Fig. 3. One of the crucial factors essential for a remodeling minimization effect is the mobility of progenitor cells. Prerequisite for the success of cell therapy is the homing and, thus, engraftment of transplanted cells into the target area. The migratory capacity of the infused cells is a major determinant of infarct remodeling, disclosing a causal effect of progenitor cell therapy on regeneration enhancement. Britten et al. first time demonstrated that the migratory capacity of transplanted progenitor cells is an independent predictor of infarct remodeling as measured by MRI-determined LE volume. ${ }^{35}$ Similarly Poole et al. showed that product potency (in terms of improvement in perfusion and LVEF) was related to the mobility of CD34 + cells in an SDF-1 gradient. ${ }^{26}$ Furthermore, they showed that cell mobility towards the chemokine gradient declined over time following bone marrow harvest, with a median $57 \%$ decrease between 24 and 48 hours post-harvest and a further $11 \%$ decline by $72 \mathrm{~h}$. Thus, administration of cells without delay after harvest may reduce the quantity of cells required for the therapeutic effect, increase the migratory capacity of the cells and enhance the therapeutic effect of cell therapy. ${ }^{36,37}$ Thus, the numbers of CD34+ 


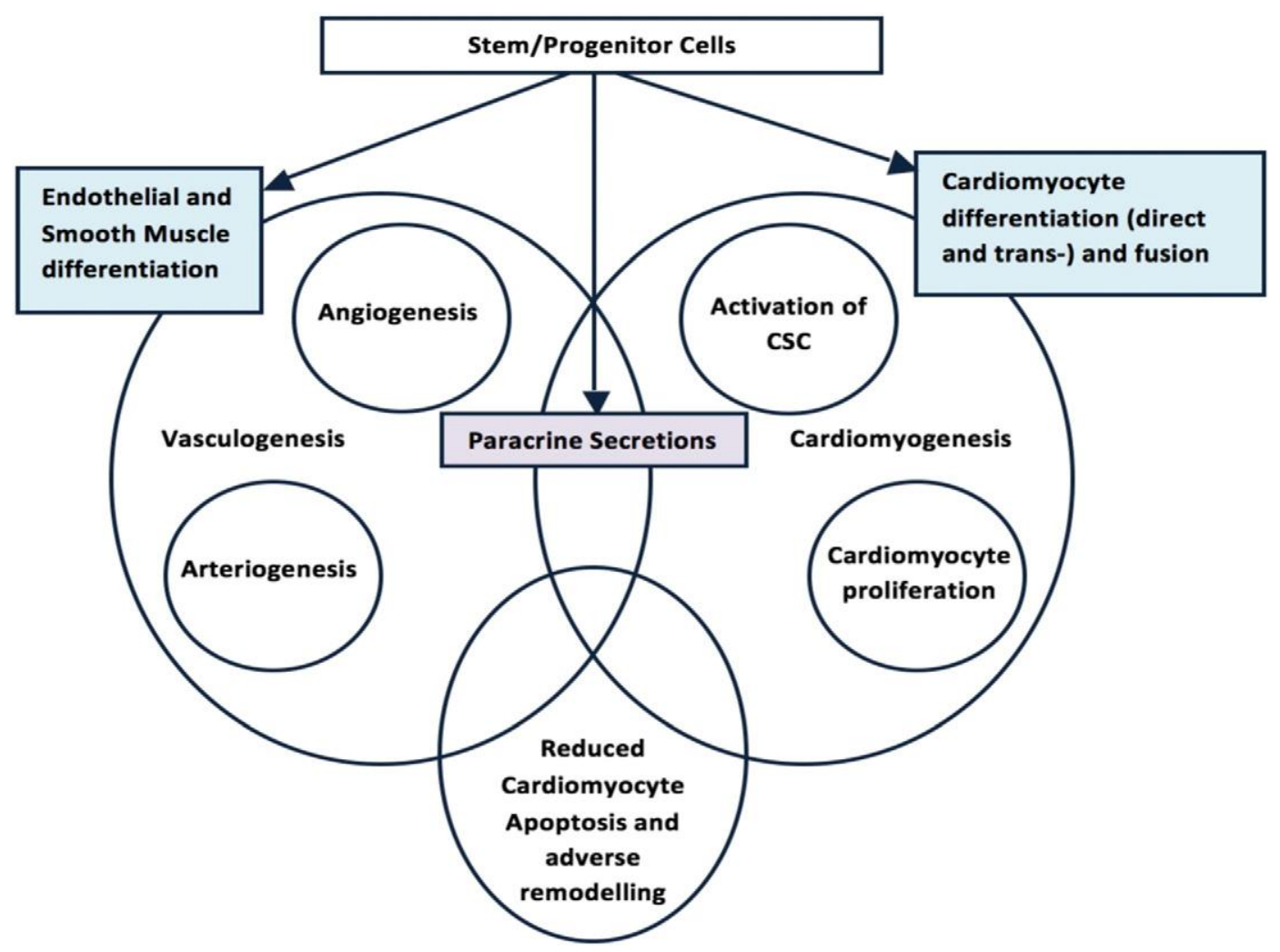

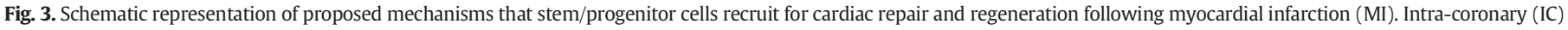
delivery of stem/progenitor cells, upregulation of CXCR4 and egress of progenitor cells from the bone marrow into the vascular bed to sustain repair and modulate inflammation.

cells, their SDF-1 mobility, and the time from harvest to infusion are all factors that appear to determine potency of the cell therapy product. Delivering the cellular product with a minimum interval lag time presents an ideal technology to the enthusiastic clinicians. Our point-of-care technology can accomplish the complete procedure, from bone marrow harvest to infusion, in 60-90 min, thus minimizing any impact of time and environmental effects on the therapeutic cells.

The final critical variable controlled in our treatment method is the utilization of an inherently cell friendly and endothelium safe intracoronary catheter designed to measure and control the impact of pressure, shear, and surface chemistry within the delivery lumen on the therapeutic cells. Most studies analyze the cellular viability, and fewer analyze the cellular potency of the cells pre- and post-traversing the catheter. The lack of attention given in previous cardiac therapies to the impact of such physical conditions has on the infuscate, again opened the possibilities for highly unpredictable results. Our procedure utilizes a proprietary controlled process and device which minimizes and measures these critical variables.

\section{Conclusion and future directions}

In brief, we have developed a rapid bedside (point-of-care) method and technology for the aspiration, processing and infusion of BMCePC, as an adjuvant treatment in cardiovascular disorders - specifically primary acute myocardial infarction. The methodology employs a heparin-free bone marrow aspiration along with a user independent automated processing system to extract BMCePC rapidly in a point-ofcare controlled environment. The technique helps overcomes few of the current problems encountered in the field of cardiac regenerative medicine and provides the first combination product (both device and therapeutic cells) for a rapid point-of-care technology of aspirating, isolating, and delivering of bone marrow derived progenitor cells and factors from the patient's own body. Many experimental studies and clinical trials have proven the feasibility, safety, and efficacy of cell- based therapy, including our case study, however, the mechanisms underlying the regeneration of damaged cardiac tissues by progenitor cells are not fully understood. There are many issues related to the use of cell therapy, including the eligibility of patients, optimal timing of cell transfer, dosage, the best way to prepare the cells, method of cell administration, and selection of the correct type of cells, that remain to be solved. Thus, large scale randomized-controlled trials are warranted to assess the clinical effects of the BMCePC administration in patients with acute myocardial infarction with an optimal delivery systems.

\section{Acknowledgments}

We would like to acknowledge the scientific, clinical, and imaging team from the medical staffs at Fortis Escort Heart Institute (New Delhi), Cedars Sinai medical Center (Los Angeles), and Transcontinental cardiovascular Core lab (TC3) (San Francisco \& Los Angeles).

\section{References}

1. Ponemone V, Bukhari S, Harris K, et al. Safety and Efficacy of Autologous Bone Marrow Mononuclear Cells in Patients with Severe Critical Limb Ischemia; 2016 [Manuscript under publication].

2. Ponemone V, Gulati R, Sivilotti M, et al. Intrathecal administration of autologous bone marrow cells with $10 \%$ hematocrit - RBCs are clinically safe. Annual ISCT Meeting. Cytotherapy 2012;15(4):1157-1287 [Poster 116, Seattle, WA].

3. Ponemone V, Gulati R, Sharma A, et al. Autologous bone marrow derived stem cell graft facilitates remodelling of non-union fractures. Annual ISCT Meeting. Cytotherapy 2012;Vol 15(4):1157-1287 [Poster 191, Seattle, WA].

4. Global Status Report on Non-Communicable Diseases 2010. Geneva: World Health Organization; 2011.

5. Global Atlas on Cardiovascular Disease Prevention and Control. Geneva: World Health Organization; 2011

6. Fisher SA, Doree C, Brunskill SJ, et al. Bone marrow stem cell treatment for ischemic heart disease in patients with no-option of revascularization: a systematic review and meta-analysis. PLoS One 2013;8e64669.

7. Clifford DM, Fisher SA, Brunskill SJ, et al. Long-term effects of autologous bone marrow stem cell treatment in acute myocardial infarction: factors that may influence outcomes. PLoS One 2012;7e37373. 
8. Tuty Kuswardhani RA, Soejitno A. Bone marrow-derived stem cells as an adjunctive treatment for acute myocardial infarction: a systematic review and meta-analysis. Acta Med Indones-Indones J Intern Med 2011;43:168.

9. Williams AR, Hare JM. Mesenchymal stem cells: biology, patho-physiology, translational findings, and therapeutic implications for cardiac disease. Circ Res 2011;109: 923.

10. Madonna R, Geng YJ, Caterina RD. Adipose tissue-derived stem cells: characterization and potential for cardiovascular. Arterioscler Thromb Vasc Biol 2009;29:1723.

11. Hayashi E, Hosoda T. Therapeutic application of cardiac stem cells and other cell types. Biomed Res Int 2013736815.

12. Burdon TJ, Paul A, Noiseux N, et al. Bone marrow stem cell derived paracrine factors for regenerative medi-cine: current perspectives and therapeutic potential. Bone Marrow Res 2011:207326.

13. Foley T, Mankad S, Anavekar N, et al. Measuring left ventricular ejection fraction techniques and potential pitfalls. Eur Cardiol 2012;8:108.

14. Tonge C, Fernandez R, Harbinson M. Current is-sues in nuclear cardiology. Br J Radiol 2008;81:270

15. Takahashi M. Role of the SDF-1/CXCR4 system in myocardial infarction. Circ J 2011;74:418.

16. Prokoph S, Chavakis E, Levental KR, et al. Sustained delivery of SDF-1a from heparin-based hydrogels to attract circulating proangiogenic cells. Biomaterials 2012;33:4792.

17. Penn MS. Importance of the SDF-1:CXCR4 axis in myocardial repair. Circ Res 2009;104:1133.

18. Seeger FH, Rasper T, Fischer A, et al. Heparin disrupts the CXCR4/SDF-1 axis and impairs the functional capacity of bone marrow-derived mononuclear cells used for cardio-vascular repair. Circ Res 2012;111:854.

19. De Vriese S, Mortier S, Lameire N, et al. Non anti-coagulant effects of heparin: implications for animal models of peritoneal dialysis. Perit Dial Int 2001;21(Supplement 3).

20. Kapur NK, Shenoy C, Yunis AA, et al. Distinct effects of unfractionated heparin versus bivalirudin on circulating angiogenic peptides. PLoS One 2012:e34344.

21. Williams RT, Damaraju LV, Mascelli MA, et al. Anti-platelet factor $4 /$ heparin anti-bodies: an independent predictor of 30-day myocardial infarction after acute coronary ischemic syndromes. Circulation 2003;107:2307.

22. Seeger FH, Tonn T, Krzossok N, et al. Cell isolation procedures matter: a comparison of different isolation protocols of bone marrow mononuclear cells used for cell therapy in patients with acute myocardial infarction. Eur Heart J 2007;28:766.

23. Assmus B, Tonn T, Seeger F, et al. Red blood cell contamination of the final cell product impairs the efficacy of autologous bone marrow mononuclear cell therapy. J Am Coll Cardiol 2010;55:13.

24. Traverse JH, Henry TD, Pepine CJ, et al. Effect of the use and timing of bone marrow mononuclear cell delivery on left ventricular function after acute myocardial infarction. JAMA 2012;308(22):e1

25. Posel C, Moller K, Frohlich W, et al. Density gradient centrifugation compromises bone barrow mononuclear cell yield. PLoS One 2012;7e50293.

26. Poole JC, Quyyumi AA. Progenitor cell therapy to treat acute myocardial infarction: the promise of high-dose autologous CD34+ bone marrow mononuclear cells. Stem Cells Int 201365848.

27. Gyöngyösi M, Wojakowski W, Lemarchand P, et al. Meta-analysis of cell-based CaRdiac stUdiEs (ACCRUE) in patients with acute myocardial infarction based on individual patient data. Circ Res 2015;116:1346-1360.

28. Surder D, Manka R, Lo Cicero V, et al. Intracoronary injection of bone marrow-derived mononuclear cells early or late after acute myocardial infarction effects on global left ventricular function. Circulation 2013;127:1968-1979.

29. Balsam LB, Wagers AJ, Christensen JL, et al. Hematopoietic stem cells adopt mature hematopoietic fates in ischemic myocardium. Nature 2004:428:668-673.
30. Murry CE, Soonpaa MH, Reinecke $\mathrm{H}$, et al. Hematopoietic stem cells do not transdifferentiate into cardiac myocytes in myocardial infarcts. Nature 2004;428: 664-668.

31. Yeh ET, Zhang S, Wu HD, et al. Trans-differentiation of human peripheral blood CD34 + enriched cell population into cardiomyocytes, endothelial cells, and smooth muscle cells in vivo. Circulation 2003;108:2070-2073.

32. Boyle AJ, Whitbourn R, Schlicht S, et al. Intra-coronary high-dose CD34+ stem cells in patients with chronic ischemic heart disease: a 12-month follow-up. Int J Cardiol 2006;109:21-27.

33. Kocher AA, Schuster MD, Szabolcs MJ, et al. Neovascularization of ischemic myocardium by human bone-marrow-derived angioblasts prevents cardiomyocyte apoptosis, reduces remodeling and improves cardiac function. Nat Med 2001;7:430-436.

34. Lipinski MJ, Biondi-Zoccai GGL, Abbate A, et al. Impact of intracoronary cell therapy on left ventricular function in the setting of acute myocardial infarction: a collaborative systematic review and meta-analysis of controlled clinical trials. J Am Coll Cardiol 2007;50:1761-1767.

35. Britten MB, Abolmaali ND, Assmus B, et al. Infarct remodeling after intracoronary progenitor cell treatment in patients with acute myocardial infarction (TOPCARE-AMI): mechanistic insights from serial contrast-enhanced magnetic resonance imaging. Circulation 2003;108:2212-2218.

36. Yao K, Huang R, Sun A, et al. Repeated autologous bone marrow mononuclear cell therapy in patients with large myocardial infarction. Eur J Heart Fail 2009;11:691-698.

37. Brunskill SJ, Hyde CJ, Doree CJ, et al. Route of delivery and baseline left ventricular ejection fraction, key factors of bone-marrow derived cell therapy for ischaemic heart disease. Eur J Heart Fail 2009;11:887-896.

38. Jeevanantham V, Butler M, Saad A, et al. Adult bone marrow cell therapy improves survival and induces long-term improvement in cardiac parameters. Circulation 2012;126:551-568.

39. Malliaras K, Kreke M, Marbán E, et al. The stuttering progress of cell therapy for heart disease. Clin Pharmacol Ther 2011;90(4):532-541.

40. Schachinger V, Erbs S, Elsasser A, et al. Improved clinical outcome after intracoronary administration of bone-marrow derived progenitor cells in acute myocardial infarction: final 1-year results of the REPAIR-AMI trial. Eur Heart J 2006;27(23):2775-2783.

41. Lunde K, Solheim S, Aakhus S, et al. Intracoronary injection of mononuclear bone marrow cells in acute myocardial infarction. N Engl J Med 2006;355(12):1199-1209.

42. Assmus B, Fischer-Rasokat U, Honold J et al Trans-coronary transplantation of functionally competent BMCs is associated with a decrease in natriuretic peptide serum levels and improved survival of patients with chronic postinfarction heart failure: results of the TOPCARECHD registry. Circ Res 2007;100(8):1234-1241.

43. Strauer BE, Yousef M, Schannwell CM. The acute and long-term effects of intracoronary stem cell transplantation in 191 patients with chronic heart failure: the STAR-heart study. Eur J Heart Fail 2010;12(7):721-729.

44. Meyer GP, Wollert KC, Lotz J, et al. Intracoronary bone marrow cell transfer after myocardial infarction: 5-year follow-up from the randomized-controlled BOOST trial. Eur Heart J 2009;30(24):2978-2984

45. Tendera M, Wojakowski W, Ruzyllo W, et al. Intracoronary infusion of bone marrow-derived selected CD34(+) CXCR4 $(+)$ cells and non-selected mononuclear cells in patients with acute STEMI and reduced left ventricular ejection fraction: results of randomized, multicentre myocardial regeneration by intracoronary infusion of selected population of stem cells in acute myocardial infarction (REGENT) trial. Eur Heart J 2009;30(11):1313-1321.

46. Makkar RR, Smith RR, Cheng K, et al. Intracoronary Cardiosphere-derived cells for heart regeneration after myocardial infarction (CADUCEUS): a prospective, randomised phase 1 trial. Lancet 2012;379(9819):895-904

47. Bolli R, Chugh AR, D'Amario D, et al. Cardiac stem cells in patients with ischaemic cardiomyopathy (SCIPIO): initial results of a randomized phase 1 trial. Lancet 2011;378(9806):1847-1857. 